\title{
Germanica
}

\section{Aristophane et Shakespeare en bandes dessinées : Ralf König et les réécritures de classiques dans Lysistrata et Jago}

Aristophanes and Shakespeare in comics : Ralf König's rewriting of literary classics in Lysistrata and Jago.

Aristophanes und Shakespeare als Comics : Ralf Königs Neufassungen von klassischer Literatur in Lysistrata und Jago.

Éric Chevrel

\section{OpenEdition}

\section{Journals}

Édition électronique

URL : http://journals.openedition.org/germanica/1132

DOI : $10.4000 /$ germanica. 1132

ISSN : 2107-0784

\section{Éditeur}

Université de Lille

\section{Édition imprimée}

Date de publication : 31 décembre 2010

Pagination : 145-159

ISBN : 9782913857261

ISSN : 0984-2632

\section{Référence électronique}

Éric Chevrel, «Aristophane et Shakespeare en bandes dessinées : Ralf König et les réécritures de classiques dans Lysistrata et Jago », Germanica [En ligne], 47 | 2010, document 10, mis en ligne le 01 décembre 2012, consulté le 06 octobre 2020. URL : http://journals.openedition.org/germanica/1132 ; DOI : https://doi.org/10.4000/germanica.1132

Ce document a été généré automatiquement le 6 octobre 2020.

(c) Tous droits réservés 


\title{
Aristophane et Shakespeare en bandes dessinées : Ralf König et les réécritures de classiques dans Lysistrata et Jago
}

\author{
Aristophanes and Shakespeare in comics : Ralf König's rewriting of literary \\ classics in Lysistrata and Jago. \\ Aristophanes und Shakespeare als Comics : Ralf Königs Neufassungen von \\ klassischer Literatur in Lysistrata und Jago.
}

\section{Éric Chevrel}

L'univers habituel de Ralf König se situe depuis ses débuts dans les années 1980 presque toujours à l'ère contemporaine, avec des albums qui retracent, sous la forme de comédies de mœurs, la vie sentimentale de personnages homosexuels, et des séries qui s'attachent à l'évolution ou aux tribulations de héros qu'on retrouve d'album en album, comme dans les trois tomes de Konrad und Paul, prolongés d'une ramification supplémentaire dans la trilogie Bullenklöten, Superparadise et Sie dürfen sich jetzt küssen. C'est aussi le cas de Der bewegte Mann, suivi de Pretty Baby, et de Kondom des Grauens continué par Bis auf die Knochen. On retrouve là une caractéristique de la bande dessinée et de la littérature populaire en général: le goût pour des personnages récurrents aux aventures toujours renouvelées. C'est sur quoi se fondent justement les critiques qui dénoncent un art qu'ils ne reconnaissent pas comme tel, car il ne saurait qu'exploiter une recette, un filon, et sa production matérielle massive, en série, irait inévitablement de pair avec une esthétique de la répétition et de la reproduction, dépourvue d'imagination et de créativité véritablement artistique. Ces attaques sont similaires à celles qui ont touché un autre art visuel récent, né également à la fin du xixe siècle, le cinéma, et auquel la bande dessinée est souvent associée ${ }^{1}$, à la fois par les aspects techniques d'une narration en images et par son caractère massif et populaire. 
Or ce reproche à l'encontre de la bande dessinée, forme qui a été souvent méprisée pour sa prétendue vulgarité ou cantonnée à la littérature pour enfants (elle aussi plus jugée sommairement qu'analysée) oublie largement que la «vraie» ou "grande» littérature n'est pas constituée exclusivement de créations originales dues au prétendu génie d'un auteur, mais qu'elle se nourrit aussi de reprises, tant internes qu'externes, de "continuations ", comme dans le Simplicissimus de Grimmelshausen ou entre le Werther de Goethe et Lotte in Weimar de Th. Mann, de réécritures comme dans Die neuen Leiden des jungen $W$. d'Ulrich Plentzdorf, et qu'elle est prise dans une intertextualité dont l'analyse révèle de nouvelles dimensions de sens. La bande dessinée est donc, ne serait-ce qu'à cet égard, une forme parfaitement littéraire, et son potentiel intertextuel est d'autant plus important qu'en raison de son apparition tardive à la fin du xixe siècle elle constitue une forme récente et mixte, qui est du fait de sa position surplombante à même de retraiter une vaste et double culture, aussi bien textuelle que visuelle.

L'œuvre de Ralf König² est riche de multiples aspects, sur le plan éthique par l'engagement contre l'homophobie, combattue par le rire et l'ironie, et aussi plus récemment contre l'obscurantisme religieux avec Archetyp et Prototyp (2008 et 2009), tout comme sur le plan esthétique par la qualité des dialogues et l'expressivité du dessin, mais elle s'inscrit également dans une relation d'intertextualité avec la "grande " littérature en particulier dans deux albums. Lysistrata (1987) et Jago (1998) sont en effet les premiers textes de König à se dégager de l'ancrage dans l'Allemagne contemporaine, et surtout ils se présentent ouvertement, dès le titre, comme des réécritures de classiques, de pièces d'Aristophane et de Shakespeare, Lysistrate et Othello.

La reprise et la transformation de textes appartenant à la littérature universelle n'est pas rare en bande dessinée, ce qui la rapproche du roman, certes moins récent, mais qui dans l'histoire littéraire s'est imposé face à un système des genres bien établi dans une littérature européenne marquée par Aristote, entre tragédie, comédie et épopée. Le roman vient ainsi prendre la place vacante d'une forme ou d'un détournement comique de l'épopée, avant de parvenir à son autonomie esthétique et à sa propre tradition, amplement multiforme. La bande dessinée ne se limite certes pas à une dimension strictement comique, mais elle se développe aussi en s'appuyant sur d'autres genres littéraires antérieurs, en les retravaillant et en lui conférant ses spécificités graphiques. Un exemple en est pour l'espace germanophone le transfert de la matière de Faust, avec le dyptique des auteurs français Michel Crespin (dessin) et Karel Dhoyen (scénario), qui avec Faust. Le remords de Dieu et Faust d'Heidelberg. L'Étudiant ${ }^{3}$ reprennent des éléments du «Volksbuch » avant Marlowe et Goethe pour proposer la préhistoire du personnage, avant qu'il ne devienne un mythe. On retrouve là une tendance de la bande dessinée au décentrement, à se faire sa place en marge de la littérature dominante, mais en étant aussi capable de pratiquer un retour aux sources (du "Volksbuch») éclairant, usant ainsi d'un procédé parfaitement littéraire, qui est de surcroît dans ce cas en accord avec la période traitée ${ }^{4}$. En Allemagne même, en raison de son poids culturel, c'est surtout le Faust de Goethe qui a fait l'objet d'une réadaptation en bande dessinée, par exemple par Flix, qui donne sa propre version contemporaine de Faust ${ }^{5}$, où le héros est chauffeur de taxi, et Gretchen, d'origine turque. L'édition souligne déjà extérieurement la dimension ludique avec cette référence littéraire écrasante dans les pays germanophones : la couverture de l'album, malgré ses dimensions usuelles, imite par la couleur et le graphisme du titre la 
collection Reclam, jouant par là avec son appartenance à la culture scolaire et universitaire. Cette version de Flix est en même temps une auto-réécriture, puisqu'il revient en fait à sa toute première publication, déjà sous le signe de Goethe, mais sous un titre plus provocateur, Who the fuck is Faust ? ${ }^{6}$ qui n'aurait peut-être pas permis à l'époque sa prépublication dans la Frankfurter Allgemeine Zeitung comme la nouvelle version de 2010, et comme justement Prototyp puis Archetyp de König, parus dans le même quotidien.

Mais il faut noter que cette "audace» ou «l'irrespect » de la bande dessinée envers l'héritage de Goethe ne lui est pas propre: à côté de la préhistoire "sérieuse " de Crispin et Dhoyen, de la réécriture ludique de Flix, il a existé dès le xixe siècle une continuation littéraire humoristique avec Friedrich Theodor Vischer, qui fait paraître en 1862 Faust. Der Tragödie dritter Theil in drei Acten. Treu im Geiste des zweiten Theils des Göthe'schen Faust.On voit ainsi que la bande dessinée est moins en rupture avec la culture classique et sa littérature qu'elle n'en déploie de nouvelles possibilités, qu'elle est aussi la continuation de la littérature par d'autres moyens. Kafka est un autre exemple de ces passerelles entre littérature et bande dessinée, avec plusieurs transpositions, comme la présentation de l'ensemble de l'œuvre par Robert Crumb et David Zane Mirowitz (1993), l'adaptation de plusieurs nouvelles par Peter Kuper (1995), de Der Proce $\beta$ par Chantal Montellier et David Zane Mirowitz (2008), et de Die Verwandlung par Horne et Éric Corbeyran (2009). On peut dire avec Monika Schmitz-Emans qu'il existe en l'espèce une affinité entre l'écriture de Kafka, qui montre les limites du langage verbal, et un art visuel qui peut d'autant mieux illustrer ces échecs, tout en étant un médium permettant de représenter l'interpénétration entre rêve et réalité propre aux récits de Kafka, sans forcément être d'accord avec la thèse d'une culture visuelle qui serait l'apanage des États-Unis ${ }^{7}$. C'est en effet beaucoup négliger la richesse de la bande dessinée européenne, en particulier francophone et italienne, et ignorer les réalisations germanophones, ce qui constitue d'ailleurs un trait général des études allemandes sur la bande dessinée, qui tendent à privilégier abusivement ses origines et évolutions américaines.

Un autre lien avec Kafka montre dans l'autre sens l'influence de la littérature sur la bande dessinée: Art Spiegelman a pu voir dans le récit de Kafka Josefine, die Sängerin oder Das Volk der Mäuse, une source d'inspiration pour son idée de représenter les juifs par des souris dans son album saisissant sur la Shoah, Maus. ${ }^{8} \mathrm{En}$ faisant des souris les héros tragiques d'une histoire qui est aussi un témoignage autobiographique, Spiegelman réaffirme la dignité de la bande dessinée à travers une référence et un hommage au représentant le plus connu de son proche parent, le dessin animé, Mickey Mouse, que le nazisme a justement attaqué comme le symbole d'une culture "dégénérée " ${ }^{9}$. On voit ici, même à travers ces quelques exemples rapidement évoqués, que la bande dessinée possède une richesse et une subtilité intertextuelles souvent sous-estimées, notamment par des critiques qui restent prisonniers de catégories préconçues sur une forme inférieure destinée aux masses, et qui procèdent à des assimilations et généralisations, vite étendues à toute une production dont la réalité est pourtant bien plus diversifiée ${ }^{10}$.

Inversement, l'ouverture de la littérature à la dimension graphique débouche sur de nouvelles possibilités expressives et narratives, comme l'illustre en un double sens le recueil d'hommage pour le 175e anniversaire de la naissance du «père » de la bande 
dessinée pour l'espace germanophone, Wilhelm Busch und die Folgen, où l'on retrouve notamment des contributions dessinées de Volker Reiche («Strizz »), Flix, Anike Hage (qui représente une bande dessinée allemande inspirée des mangas) et Ralf König ${ }^{11}$. Le titre lui-même constitue une allusion littéraire et humoristique à l'essai de Karl Kraus « Heine und die Folgen » (1910), à la différence fondamentale que Wilhelm Busch n'est pas considéré ici comme le début d'une décadence de l'écriture comme l'incarne aux yeux de Kraus un Heine faisant descendre la littérature vers le style journalistique, mais bien d'un nouvel art, ou à tout le moins d'un art renouvelé, et où l'humour joue justement un rôle non négligeable.

Le caractère ludique est ainsi l'un des aspects les plus visibles des réécritures que sont Lysistrata et Jago, qui exploitent à l'échelle d'un album entier la citation de la grande littérature et son détournement, techniques utilisées déjà bien avant plus ponctuellement dans des "classiques» de l'école franco-belge. C'est le cas du Jesse James de Morris et Goscinny (1969), où le frère du célèbre bandit, Frank, s'exprime systématiquement par des citations de Shakespeare d'une extrême banalité. Dans Astérix chez les Belges d'Uderzo et Goscinny (1979), la bataille finale entre Belges et Romains, référence à Waterloo, adapte dans les encadrés du narrateur des extraits du poème "L'expiation", issu des Châtiments de Victor Hugo (1853), imitant tout d'abord son style épique, avant de créer une chute par un retour à la langue parlée qui va de pair avec le refus de l'héroïsme et du sacrifice, du culte du grand homme, à l'encontre du mythe napoléonien incarné ici par César. Semblablement, König procède à une démythisation de Shakespeare en le faisant dans Jago d'emblée descendre de son piédestal, en l'occurrence tomber de cheval, le "grand auteur » étant montré ivre dans les premières pages, urinant sur un pal et signant des autographes rimés vulgaires $^{12}$. L'introduction d'érotisme et de sexualité, très présents dans tous les albums de König, est aussi un élément qui contribue à tourner en dérision les genres établis qui servent de base au travail de réécriture ${ }^{13}$.

Sur le plan formel, le jeu avec la référence littéraire et la critique implicite de ses règles apparaît dans le découpage des deux albums en un nombre d'« actes " qui marque une inflation peu conventionnelle : sept pour Lysistrata, neuf pour Jago, ce qui souligne à la fois une forme de fidélité au genre théâtral, et la liberté dans son traitement par la bande dessinée. On retrouve cette démarche dans De cape et de crocs d'Ayroles et Masbou (neuf albums et autant d'actes parus depuis 1995, série en cours chez Delcourt), qui se déroule au xvire siècle et qui multiplie les références au théâtre, surtout à Molière, tout en suivant un principe d'accumulation d'aventures, de Venise à la lune, qui ressortit à un autre genre du xvire siècle, le roman picaresque.

Lysistrata et Jago reprennent néanmoins des éléments de leur textes-sources qui permettent d'assurer la nécessaire reconnaissance par le lecteur, et qui permettra aussi de mieux percevoir le décalage avec ce qu'apporte la bande dessinée à l'original : dans Jago, c'est la citation textuelle de drames et de sonnets de Shakespeare ${ }^{14}$ qui remplit cette fonction, tandis que Lysistrata opère davantage par la citation visuelle, ou la reprise d'une situation dramatique ${ }^{15}$. Le travestissement ludique qui met à distance le texte original passe aussi par des procédés assez banals, comme les anachronismes marqués, qui émaillent surtout Lysistrata, où l'on regarde "Dynasty" à la télévision ${ }^{16}$, et où les noms des personnages reposent sur des jeux de mots: sur le modèle d'un personnage d'Aristophane, Myrrhine, König crée par exemple Klementine et Lawine ${ }^{17}$, qui sont aussi des allusions au type de noms des Gauloises dans Astérix 
(que ce soit en français ou en traduction: Bonnemine, femme d'Abraracourcix, s'appelle en allemand Gutemiene) ; les hommes portent des noms tout aussi parlants, comme Hepatitos, Incognitos, Ohropax, Thermos, Kleenax ${ }^{18}$, mais il faut noter qu'il s'agit moins d'un travestissement que d'une continuité à l'intérieur du registre comique sous le signe de la surenchère avec Aristophane, qui se servait déjà de noms expressifs et révélateurs. Jago recourt en revanche beaucoup moins à ce procédé et est en comparaison beaucoup plus "réaliste", ce qui correspond d'ailleurs aussi à la pratique graphique, plus riche en décors et en détails. Mais Jago travestit malgré tout partiellement Othello en tragi-comédie ${ }^{19}$, en ce qu'il donne une fin positive à l'album, Tom Poope et Gronzo Granato échappant à la mort ${ }^{20}$ et se réfugiant en Afrique pour y vivre heureux, au moins un temps.

Si ces éléments humoristiques constituent surtout des signaux extérieurs de parodie ${ }^{21}$, les changements les plus notables qu'apporte König aux deux pièces sont surtout d'ordre thématique, associant aux sujets de Shakespeare et d'Aristophane ses propres thèmes de prédilection, la représentation du monde homosexuel et la lutte contre l'hostilité que la société leur renvoie. König fait ainsi entrer son propre univers dans les deux textes, y compris ses personnages et des types de relations: Poope, membre historique de la troupe du Globe Theatre dans Jago, présente beaucoup de ressemblances avec Paul dans la série Konrad und Paul, et sa liaison avec l'imposant Noir Gronzo rappelle pour une bonne part celle avec Ramon, musculeux ouvrier espagnol dans Bullenklöten, tous deux s'exprimant dans une langue simplifiée et fautive. L'intégration de l'univers propre à l'auteur moderne, ses autoréférences dans les textes classiques contribuent déjà à leur détournement. Mais König s'appuie en même temps sur deux textes qui présentent déjà des affinités certaines avec le thème de la sexualité : les ressorts dramatiques d'Othello et de Lysistrate reposent sur les relations amoureuses et sexuelles, la jalousie du Maure attisée par un Iago qui se sert aussi de sa propre femme et d'une courtisane, Bianca, pour manipuler son monde, la grève du sexe par les femmes chez Aristophane. Le traitement langagier de la sexualité est aussi proche des dialogues et du dessin de König, qui nomme les choses par leur nom et les montre sans fausse pudeur: surtout Aristophane, mais aussi Shakespeare recourent fréquemment à des jeux de mots crus et à des allusions sexuelles qui ont été mis en lumière par des retraductions récentes, dans le texte ou en notes, et qui s'éloignent salutairement d'un souci de décence « littéraire » qui ne figure pas dans les textes originaux ${ }^{22}$.

Dans un cadre qui associe érotisme et exotisme (la Grèce dans Lysistrata, l'Afrique dans Jago, avec le récit des origines de Gronzo et les ambiguïtés de l'idylle finale avec Poope ${ }^{23}$ ), l'apport essentiel de König consiste naturellement à greffer les amours homosexuelles sur l'action des deux pièces : tous les membres de la troupe du Globe Theatre sont homosexuels, et l'album fait principalement le récit des machinations $\mathrm{du}$ comédien Gus (Augustine) Philipps, autre personnage ayant réellement existé, contre son collègue Poope pour avoir les faveurs de Gronzo, le colosse noir qui correspond physiquement à l'Othello de Shakespeare, mais dont la fonction se trouve largement modifiée. Gronzo n'appartient pas au monde du théâtre, et ce n'est pas sa jalousie qui constitue le ressort de l'histoire, mais bien celle qu'il suscite chez Philipps envers Poope, et qui se double d'une jalousie professionnelle, car dans l'album, Shakespeare a prévu de donner le rôle de Iago à Poope, alors que Philipps estime être meilleur comédien. Dans la configuration des personnages, Philipps correspond au Iago de l'original élisabethain, voulant détruire le couple Othello-Desdémone, représenté ici 
par le couple Gronzo-Poope. Chez Shakespeare, Iago cherche à reconquérir la charge d'officier qu'Othello a attribuée à Cassio en convainquant le premier que sa femme Desdémone le trompe avec Cassio ; König condense l'intrigue en créant un triangle exclusivement masculin et homosexuel, où pour Philipps le rival amoureux est identique au rival professionnel. Dans Lysistrata, König conserve la thématique sexuelle de la grève des femmes, mais il lui ajoute une suite qui relance l'action : les homosexuels d'Athènes et de Sparte profitent de cette situation de désespérance sexuelle des hommes pour convertir, avec un succès provisoire, la population masculine à l'homosexualité, en persuadant les états-majors que l'«homosexualité forcée " est le seul moyen pour les soldats d'être de nouveau en état de combattre l'ennemi dans la guerre du Péloponnèse. La réécriture de la pièce d'Aristophane recèle ainsi une composante de continuation, qui complexifie la situation en introduisant une homosexualité qui n'est d'ailleurs pas que masculine : les deux femmes d'Athènes et de Sparte qui lancent l'idée de la grève du sexe, Lysistrata et Lampito, qui conservent leur nom dans l'album de König, forment, à la différence d'Aristophane, un couple de lesbiennes ${ }^{24}$, tout comme dans Jago Anne Gaulswallow et Hippolyta (dont les noms contiennent en allemand et en grec le terme " cheval » qui correspond à leur métier, équarisseuses de chevaux). La réécriture des deux pièces passe donc par l'introduction générale de l'homosexualité, essentiellement masculine, ce qui induit un décentrement, en mettant au cœur de l'action une population homosexuelle sinon "marginale », qui va même pour un temps être dominante dans Lysistrata.

De ce fait, les deux albums comportent et réinterprètent aussi une dimension politique déjà présente dans les deux textes originaux : représentée en 411 avant J.-C., en pleine guerre du Péloponnèse, la pièce d'Aristophane prend position pour la paix, et la quasiabsence d'attaques directes envers des personnes réelles, contrairement à ses habitudes littéraires (comme contre Socrate dans les Nuées), incite à voir dans la lutte contre la guerre, malgré le mode comique, un appel authentique à la paix entre les deux adversaires. Or cette paix improbable passe par une remise en cause de préjugés « nationaux » analogue à la révision de la domination biologiquement « naturelle » des hommes sur les femmes unanimement acceptée dans l'Antiquité grecque, par la critique d'un bellicisme inhérent au machisme et au culte masculin de l'héroïsme. Dans Othello, Shakespeare fait un usage comparable du contre-emploi, en faisant jouer, au début d'un xvire siècle peu tolérant envers les autres "races ", le rôle d'un personnage noble et généreux, même s'il est aveuglé par la jalousie, par un Maure noir, tandis que c'est un Chrétien blanc qui incarne un machiavélisme sanglant. König reprend ce décentrement en le transposant aux homosexuels, qui se retrouvent au "Club Adonis " pour y faire le même diagnostic que les femmes sur cette " guerre absurde $»^{25}$, et qui sont eux-mêmes soumis au regard méprisant de la majorité de la population. Jago politise encore davantage le statut des homosexuels en ce qu'il est rappelé à plusieurs reprises que la Londres de 1603, où va être joué Othello, voit les "sodomites" poursuivis, torturés, exécutés ${ }^{26}$. Les situations de marginalité du Maure Othello dans une société chrétienne, ou des femmes dans la société patriarcale grecque, sont ainsi transférées à la communauté homosexuelle, non sans nuances critiques: le général Incognitos est un partisan de la guerre tout en étant homosexuel, et la plupart des comédiens homosexuels du Globe Theatre tiennent à admirer la plastique impeccable du bourreau Geoffrey Stryker, alors même qu'il décapite aussi des «sodomites" (et qu'il en est lui-même un). 
Dans les deux cas, König reprend la méthode de la déstabilisation des idées préconçues sur les autres races ou les femmes pour mettre en scène son monde homosexuel, la bande dessinée apparaissant par ses origines humoristiques comme une forme qui se prête particulièrement au jeu sur les références littéraires, mais aussi à la valorisation de la «marginalité » qu'elle s'est vu elle-même attribuer dans le système des arts. Cela n'empêche pas pour autant la réflexion sur la condition homosexuelle et humaine en général, comme le montrent les fins respectives de Lysistrata et de Jago : Lysistrata constate avec Lampito l'échec de son plan, détourné par les homosexuels qui sont parvenus à convertir les Grecs à l'homosexualité, provoquant l'abandon du pouvoir par les femmes, qui retournent précipitamment vers leurs hommes, scellant par contrecoup l'échec du plan de conversion homosexuelle. À Lampito qui se demande pourquoi les dieux ont bien pu créer un tel monde où les femmes aiment des hommes qui aiment faire la guerre et les opprimer, Lysistrata rappelle que le père de tous les dieux, Zeus, est justement un homme (" 'n Kerl»), sur quoi retentit la voix de Zeus, qui a le dernier mot, machiste, de la pièce ${ }^{27}$. König joue ainsi également avec la pratique du Deus ex machina, mais il fait s'achever "sa " pièce sur une note relativement pessimiste, la domination d'une masculinité hétérosexuelle sûre d'elle-même, à l'inverse d'Aristophane, dont la pièce se clôt sur une double réconciliation entre hommes et femmes, entre Athéniens et Spartiates. Ainsi, ce pied de nez final de Zeus fait que dans son Lysistrata,König ne propose pas qu'une actualisation et une continuation homosexuelle qui resteraient strictement à l'intérieur du champ comique de la pièce d'Aristophane, mais en nuance la tonalité comique. De même, Jago met à distance la pure tragédie, entrant dans la tragi-comédie par sa fin heureuse, tout en y instillant une touche mélancolique, car malgré sa nouvelle vie en Afrique aux côtés de Gronzo, Poope aspire à la dernière page à revenir en Angleterre.

Dans les deux albums, il est remarquable que König ait choisi le théâtre pour ses premières expérimentations intertextuelles avec la "grande » littérature, en usant de dispositifs de représentation qui accentuent le caractère ludique, et qui font aussi partie de l'histoire du théatre : le jeu avec l'illusion théâtrale et les interactions entre représentation et "réalité ». En effet, la pièce d'Aristophane revue par König ne commence véritablement qu'après un bref prologue où un couple (hétérosexuel) de spectateurs (du ve siècle avant J.-C.) s'installe sur les gradins et s'adresse au lecteur, supposé faire lui aussi partie du public. Or ces deux spectateurs vont régulièrement commenter l'action, à chaque fois entre deux $\operatorname{actes}^{28}$, rappelant le caractère théâtral de l'action. Par là, König reste aussi fidèle aux spécificités du théâtre grec, en transférant dans la salle la fonction du chœur, commentateur des événements. Il est ici réduit à la forme minimale de deux personnages, un homme et une femme, qui correspondent aux deux chœurs de femmes et d'hommes qui chez Aristophane redoublent l'action en s'affrontant verbalement et même physiquement, avant leur réconciliation finale, ne faisant alors plus qu'un seul chœur. Chez König aussi, l'homme et la femme reproduisent le conflit des sexes dans la salle par leurs disputes, créant même une rupture d'illusion à deux reprises, puisque les comédiens sur la scène délaissent un instant la pièce qu'ils jouent pour s'adresser à eux. Mais le fait que ce soit l'homme qui tienne des propos homophobes, qu'il ne réussisse pas à se faire obéir de sa femme, et que ce soit lui qui quitte le théâtre, abandonnant le terrain à sa femme, relativise en fait discrètement, sur le plan du récit-cadre, la victoire finale d'un Zeus imposant par la force verbale et la menace sa «morale » masculine et hétérosexuelle. 
Jago présente une autre variante du théâtre dans le théâtre en situant son action principalement dans une troupe de comédiens, qui sont montrés plusieurs fois en train de jouer Hamlet, où ceux qui ne sont pas sur scène ne sont pas avares en coulisse de commentaires sur le jeu de leurs collègues ou le texte de Shakespeare ${ }^{29}$, jusqu'à ce qu'inversement Gus Philipps, jouant enfin avec Iago son grand rôle, confonde sur scène lors de la première d'Othello la pièce avec la réalité de ses crimes, qu'il avoue devant le public et le roi, ce qui le mènera à sa dernière représentation, sur l'échafaud.

Ce choix du théâtre pour aborder en bande dessinée la littérature "classique » peut donc s'expliquer par les potentialités ludiques du théâtre qui en font, autant que le cinéma, un parent esthétique méconnu ou sous-estimé de la bande dessinée, alors que l'un comme l'autre reposent dans leur esthétique sur l'alliance des dialogues et du corporel, du verbal et du visuel ${ }^{30}$. On peut aussi supposer une affinité du théâtre avec le déguisement, le travestissement, qui peuvent être considérés comme un élément de la culture homosexuelle, ou souligner que cette valorisation de la théâtralité illustre la complexité et la diversité des identités humaines, la possibilité d'en posséder plusieurs, et de ne pas être réduit à un statut unique en adéquation avec les apparences et les clichés, immuable, notamment sur le plan sexuel. Mais le choix du théâtre par König peut être en fait aussi rattaché à une dimension propre aux pays de langue allemande, à la différence des références littéraires dans les pays francophones: reprendre et retravailler deux pièces de théâtre, c'est confronter la bande dessinée avec une culture et une institution théâtrale qui jouit en Allemagne et en Autriche d'une plus grande reconnaissance sociale. C'est être ainsi à la fois plus provocateur et plus ambitieux, et contribuer, par la réussite d'adaptations tout sauf serviles, à établir d'autant mieux la bande dessinée dans le système des arts, alors que dans les pays de langue allemande elle semble moins acceptée comme telle ${ }^{31}$. Avec ses versions des pièces d'Aristophane et de Shakespeare, König s'inscrirait même, délibérément ou non, pleinement dans l'histoire littéraire allemande, en incarnant un représentant tardif, et humoristique, de la grécomanie (plus du côté du vitalisme dionysiaque de Nietzsche que de l'éloge de la régularité chez Winckelmann) et de la shakespearomanie allemandes, courants artistiques et littéraires surtout vivaces aux xvIIIe et XIxe siècles.

D'un album à l'autre, König adopte une même attitude envers ses modèles textuels, associant imitation et transformation, transposition " sérieuse $»^{32}$ et jeu avec les codes des genres, qui témoigne de sa fidélité à l'univers homosexuel tout comme de sa capacité à s'adapter habilement à la matière et au style des textes-sources. Mais l'on peut aussi distinguer des évolutions notables entre les deux albums: Lysistrata repose avant tout sur le renversement supplémentaire que König adjoint à la pièce d'Aristophane, qui donne le pouvoir et la victoire aux femmes, leur faisant occuper symboliquement l'Acropole, haut lieu à la fois politique et religieux. En enrichissant le dualisme homme-femme d'une troisième composante, homosexuelle, König crée certes un rebondissement dramatique nouveau, mais il reste à l'intérieur de la structure de renversement et de provocation d'Aristophane. Malgré des anachronismes ponctuels frappants, comme la télévision ou l'oracle de Delphes prédisant la pandémie du Sida ${ }^{33}$, König est en somme fidèle à sa source, jusque dans la restitution minimale du chœur. Il actualise, enrichit et intensifie l'idée d'Aristophane, mais en en restant tributaire, König se plaçant explicitement dans la position du metteur en scène, comme le signale d'emblée le sous-titre présenté dans le prologue après la banderole annonçant «Lysistrata »: "Frei nach einer Komödie von Aristophanes in einer Inszenierung von Ralf König » [«Libre adaptation d'une comédie d'Aristophane, dans une mise en scène 
de Ralf König»]. Graphiquement, cette position d'interprète plus que d'auteur est soulignée par le relatif dépouillement dans la représentation de l'action, avec des fonds principalement blancs et des décors réduits, qui mettent davantage en valeur les dialogues et le jeu des physionomies. Si l'histoire se termine avec le machisme de Zeus, l'album se clôt véritablement sur une double page quasiment « muette » où l'on voit les acteurs saluer et les spectateurs applaudir, ce qui maintient jusqu'au bout le cadre théâtral de l'original.

En revanche, Jago fait preuve d'une plus grande autonomie esthétique, en ne restant pas soumis à la structure d'Othello, mais en combinant librement plusieurs motifs d'autres pièces de Shakespeare, ainsi que des sonnets. Le prologue montrant un Shakespeare ivre est ainsi suivi d'un autre prologue, où apparaissent comme dans Macbeth trois sorcières, dessinées par Walter Moers (un autre cas d'intertextualité), qui vont jouer les tentatrices auprès de Philipps; la nuit passée dans une forêt peuplée d'êtres fantastiques par Poope et Gronzo cite A Midsummer Night's Dream, et leur double suicide dans la crypte reprend la fin de Romeo and Juliet ${ }^{34}$. De plus, il fait du théâtre "seulement » le milieu et la profession des personnages dont il raconte l'histoire, les pages consacrées à la représentation des pièces étant finalement minoritaires. Cette histoire articulée autour de Gus Philipps, qui comme Iago dans Othello est le véritable personnage principal (ce que König entérine par son propre titre), s'éloigne davantage du texte de Shakespeare et se nourrit plus de l'interaction entre des personnages individualisés, faisant de l'album une œuvre plus personnelle, contrairement à la surenchère sur l'idée initiale d'Aristophane dans Lysistrata, qui tient en partie de l'exercice de style et de la continuation. Jago fait aussi ressortir une évolution graphique qui va de pair avec la plus grande liberté dans le retraitement des textes-sources : König fait preuve dans Jago, plus de dix ans après Lysistrata, d'une plus grande variété, créativité et maitrise dans le dessin. Il utilise bien plus de nuances de gris pour rendre aussi bien l'atmosphère pesante de la Londres de 1603 que la féerie de la nuit dans la forêt ${ }^{35}$, recourant également à un découpage en cases plus dynamique, comme lors de l'ascension du balcon par Gronzo rejoignant Poope ${ }^{36}$ (une autre citation de Romeo and Juliet), tandis que Lysistrata repose sur une distribution plus statique de cases qui possèdent un format plus régulier. L'hommage à Shakespeare que rend König dans sa courte postface, qui suit l'annexe donnant la liste des allusions et citations, est en fait largement réalisé par la bande dessinée elle-même, qui associe comme dans les pièces de Shakespeare tragique et dérision, scènes poignantes et comiques, envolées lyriques et jeux de mots. König imite ici moins Shakespeare qu'il ne le retrouve dans l'éclairage qu'il donne des passions humaines, mais avec un support artistique différent.

Lysistrata puis Jago montrent ainsi, à travers la réécriture et la transposition graphique de textes classiques, une capacité de la bande dessinée à participer à sa manière à la littérature, en potentialisant ses structures comme dans Lysistrata, en recombinant ses motifs pour déboucher sur un texte personnel comme dans Jago. Dans les deux cas, malgré des procédés comiques qui consistent aussi à forcer le trait, König donne des albums complexes, qui sont aussi bien loin de se limiter à une écriture « communautaire » qui ne serait destinée qu'à un lectorat homosexuel. Les pratiques intertextuelles de König montrent que la bande dessinée allemande est capable d'apporter sa contribution à la circulation et aux échanges entre les productions littéraires caractérisant la Weltliteratur que Goethe appelait de ses vœux ${ }^{37}$. 


\section{NOTES}

1. L'œuvre de Ralf König illustre d'ailleurs les possibilités de passage entre les deux médiums : Der bewegte Mann a été porté à l'écran en 1994 par Sönke Wortmann, Kondom des Grauens en 1996 par Martin Walz, Wie die Karnickel en 2002 par Sven Unterwaldt, Lysistrata la même année par Francesc Bellmunt en espagnol.

2. L'univers du dessinateur a fait récemment l'objet d'une première synthèse, par Xaver Rammbock [pseudonyme de Boris von Brauchitsch]: Die Welt der Knollennasen. Eine soziorhinologische Untersuchung, Hamburg, Männerschwarm, 2010. L'ouvrage constitue un écho lointain à l'étude fondatrice sur le monde de Donald Duck dans l'espage germanophone, par Grobian Gans [pseudonyme de Michael Czernich, Carl-Ludwig Reichert et Ludwig Moos]: Die Ducks. Psychogramm einer Sippe, Gräfelfing vor München, Wissenschaftliche Verlags-Anstalt zur Pflege deutschen Sinngutes im Heinz Moos Verlag, 1970.

3. Issy-les-Moulineaux, Vents d'Ouest, 1995, Paris et Casterman, 1998.

4. Pascal Noir et Michel Pfeifer : «L'enfance de Faust ou comment rajeunir un mythe (Faust en bande dessinée) ", in : Faust $20^{e}$, échos de l'ego. Le démon de Faust ou l'homme et ses démons, textes réunis et présentés par Pascal Noir, Paris, Caen, Lettres Modernes Minard, 2000, p. $274:$ « faire du neuf avec de l'ancien, n'était-ce pas déjà l'une des clefs de la littérature au $\mathrm{XVI}^{\mathrm{e}}$ siècle qui consistait, précisément, à s'inspirer au plus près des Anciens, et ce, afin de renouveler les Lettres modernes?»

5. Flix : Faust: der Tragödie erster Teil, Hamburg, Carlsen, 2010.

6. Frankfurt am Main, Eichborn, 1998.

7. Monika Schmitz-Emans : « Kafka in European and US Comics. Inter-medial and inter-cultural transfer process", in : Revue de Littérature Comparée 78/4, n³12, 2004, p. 488, à propos de Peter Kuper, Robert Crumb et Will Eisner : «their stylistically variant transformations of Kafka's stories into graphic narratives represent an exemplary dialogue of European literature and American visual culture. Using different techniques, each of them adapts Kafka's imaginary world to a world of images and means of expression, which is rooted in US-graphic storytelling» [ leurs différentes transformations des histoires de Kafka en récits graphiques, dans des styles variables, représentent un dialogue exemplaire entre la littérature européenne et la culture visuelle américaine. En recourant à des techniques différentes, chacun d'eux adapte le monde imaginaire de Kafka pour le faire entrer dans un monde d'images et de moyens d'expressions ancré dans la tradition américaine du récit en images »]. Le titre de l'article est trompeur : il n'y est en fait pas question de bandes dessinées européennes.

8. Andreas Platthaus: Im Comic vereint. Eine Geschichte der Bildgeschichte, Berlin, Alexander Fest, 1998, p. 279.

9. Axel Dunker : "„Time flies“. Mediale Selbstreflexivität in Art Spiegelmans Holocaust-Comic Maus», in : Der Holocaust und die Künste. Medialität und Authentizität von Holocaust-Darstellungen in Literatur, Film, Video, Malerei, Denkmälern, Comic und Musik, hrsg. v. Matías Martinez, Bielefeld, Aisthesis, 2004, p. 80.

10. C'est ce que fait par exemple Heinz-Peter Preußer: «Zerstörung, Rettung des Mythos im Trivialen. Über die Travestie der Tradition in Literatur und Film, in Fernsehen und Comic», in : Mythenkorrekturen. Zu einer paradoxalen Form der Mythenrezeption, hrsg. v. Martin Vöhler u. Bernd Seidensticker, Berlin, Walter de Gruyter, 2005, qui dans la partie finale consacrée aux bandes dessinées, p. 458-463, n'aborde que les super-héros américains, analysés de surcroît uniquement à travers leur passage au cinéma, délaissant les spécificités du support papier, pour conclure à un effet de remythisation créé par les bandes dessinées, qui seraient dépourvues de tout sens critique envers le mythe. 
11. Wilhelm Busch und die Folgen, Berlin, Ehapa, 2007.

12. Jago [1998], Reinbek, Rowohlt, 7. Aufl., 2008, p. 2-10. Shakespeare est aussi ridiculisé dans son obsession à nier ses tendances homosexuelles p. 95 et 152, ainsi que dans les faiblesses de l'intrigue d'une pièce qui devait à l'origine s'appeler «Öthellü - Der Türke von Istanbul», les réponses simplistes ou de mauvaise foi de Shakespeare aux objections de ses amis ou du roi

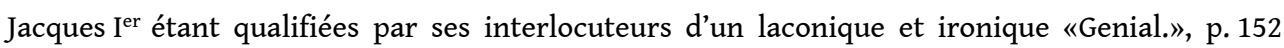
et 171.

13. On peut citer pour le domaine allemand la bande dessinée érotique de Harm Bengen, Sandra Bodyshelly (8 tomes entre 1990 et 2004), qui mêle à l'érotisme la référence au roman fantastique et d'horreur, tout comme le nom de l'hérö̈ne joue avec le peintre Sandro Botticelli et son tableau «Nascita di Venere» représentant Vénus nue, et avec le roman Frankenstein de Mary Shelley.

14. Chaque " acte " s'ouvre sur une citation encadrée placée en exergue, ce à quoi s'ajoutent les citations en situation, sur scène, lorsque les personnages jouent Hamlet ou Othello.

15. Par exemple lorsqu'une des femmes qui occupe l'Acropole veut rejoindre son mari et feint d'être enceinte en mettant un casque sous sa tunique, Lysistrata, p. 74-75, ce qui correspond exactement au texte d'Aristophane, tout comme lorsque les sexes en érection des Athéniens privés de leur femme font une bosse reconnaissable sous leur vêtement, p. 42.

16. Lysistrata [1987], Reinbek, Rowohlt, 15. Aufl., 2004, p. 101 (autre allusion à cette série télévisée p. 55 et 120). De même, l'état-major athénien se sert d'un micro, p. 61, et Kinesias prétend être membre d'un club de bowling spartiate, p. 52. Les anachronismes sont plus rares et plus discrets dans Jago, où l'on porte des vêtement de « Gavin Klyne » ou «Dolce \& Gabballa » (p. 21 et 28), ou lorsque Shakespeare imagine une première fin contemporaine après qu'Othello a étranglé Desdémone : «Jago und der Mohr werden Freunde und fahren in den Urlaub nach Ibiza. Ende » (p. 151).

17. Lysistrata, p. 59 et 60 .

18. ysistrata, p. 20, 54, 78, 89, 105.

19. Les réécritures plus ou moins comiques d'Othello ne sont pas du seul fait de la bande dessinée ou du $\mathrm{xx}^{\mathrm{e}}$ siècle: Karl Meisl écrit ainsi en 1806 pour le théâtre viennois une farce intitulée Othellerl, der Mohr von Wien oder Die geheilte Eifersucht.

20. Cet épisode est l'occasion d'un nouveau travestissement de Shakespeare, puisque König joue cette fois avec la scène de la funeste erreur dans la crypte dans Romeo and Juliet: Gronzo et Poope ratent leur suicide respectif, ce qui n'est évoqué que par un bref retour en arrière de Poope, dans Jago, p. 178.

21. La parodie est entendue ici au sens large de la reprise d'un texte avec l'introduction d'un décalage entre la matière et le registre, avec le plus souvent un passage vers le comique ou le satirique. Si l'on suit Gérard Genette : Palimpsestes. La littérature au second degré, Paris, Seuil, 1982, p. 45-46, les réécritures de König s'apparenteraient à une imitation ludique associée à une transformation « sérieuse ».

22. Aristophane : Lysistrate, übersetzt u. hrsg. v. Niklas Holzberg, Stuttgart, Reclam, 2009, par exemple p. 23 : «keine andre Hündin greift dir dann noch an die Hoden!» [« aucune autre chienne ne finira par t'attraper par les couilles »]; l'un des personnages masculins s'appelle «Kinesias von Fickingen» [Kinesias de Baiseville], p. 46. William Shakespeare : Othello. Zweisprachige Ausgabe. Deutsch v. Frank Günther, München, dtv, 1995, par exemple p. 147 : «Sehn, wie er sie besteigt?» [« (Vous voulez) voir comment il lui grimpe dessus?»]; Shakespeare joue sur l'ambiguïté des mots «sport» p. 76 ou " game » p. 84, qui désignent aussi le plaisir sexuel, ou encore «thing ", p. 136 et 138 , allusion au sexe féminin.

23. Jago, p. 33-36 et p. 178-181.

24. Elles ne sont donc pas touchées par cette grève, ce qui leur est d'ailleurs reproché par les autres femmes qui occupent l'Acropole, Lysistrata, p. 76. 
25. Lysistrata, p. 23, Hepatitos : «Dieser Krieg ist absurd! Ich sage euch, diese Heteros sind völlig beknackt!» [ “ Cette guerre est absurde ! Je vous le dis, ces hétéros sont complètement tarés »].

26. Jago, p. 155, où Anne décrit à Poope ce qui l'attend en prison; Gus Philipps se fait décapiter aussi pour son homosexualité à côté de ses crimes (il a assassiné Sam Sandwich après l'avoir poussé au meurtre du père de Poope, puis ses amis Robert et Gilbert, qui commençaient à le soupçonner), p. 176.

27. Lysistrata, p. 125 : «Richtig! Und was für einer!! Soll ich mal runterkommen und es euch beweisen?! Danach seid ihr bestimmt nicht mehr lesbisch! Hä hä hä!!!» [« Exactement ! Et quel mâle! Vous voulez que je descende et que je vous le prouve ?! Après ça, vous ne serez sûrement plus lesbiennes! Ha, ha ha!!! »].

28. Lysistrata, p. 18, 39, 46, 60, 80-81, 96-97, 126. À ces occasions, la perspective de représentation est modifiée pour faire apparaître dans le champ les spectateurs devant la scène, le dessin soulignant ainsi lui aussi la théâtralité de l'action.

29. Jago, p. 20-29 et 150-152.

30. . - Dietrich Grünewald : Comics, Tübingen, Niemeyer, 2000, p. 18, semble justement vouloir explorer ce possible lien génétique et historique entre théâtre et bande dessinée, en partant de la citation d'un "grand ancêtre » de la bande dessinée, William Hogarth, qui en 1753 se voit traiter les personnages de ses caricatures en "dramaturge" dirigeant des comédiens muets. Mais rapidement Grünewald délaisse cette piste pour se concentrer sur les «théâtres de papier » à découper du début du XIX ${ }^{\mathrm{e}}$ siècle.

31. Dietrich Grünewald: Comics, p. 69-72, souligne que la recherche sur la bande dessinée en Allemagne est encore relativement en retard, ce qu'il associe à une production autochtone plus restreinte, ainsi qu'à une lutte esthétique et «morale » encore vive contre la vulgarité supposée intrinsèque à la bande dessinée, p. 77-79. Il cite à ce propos la tentative de mise à l'index de Bullenklöten en 1995, rejetée en raison du caractère artistique l'album (p. 79).

32. C'est ainsi que Gérard Genette caractérise la relation dépourvue d'intention satirique et ludique, en donnant comme exemple la reprise du «Volksbuch» par le Doktor Faustus de Th. Mann, dans Palimpsestes, p. 43.

33. Lysistrata, p. 97. C'est le spectateur incarnant le chœur masculin, et homophobe, qui cite l'oracle.

34. Lysistrata comporte aussi un élément de recombinaison extérieure à Aristophane et à la comédie, en insérant le motif tragique, lui aussi retravaillé, d'Ædipe : profitant de l'ordre sur l'homosexualité forcée, Hepatitos parvient à séduire un certain ödipos, à qui il rappelle sa mère, et qui déteste son père (p. 87-89). Ce dernier évince Ödipos du lit de Hepatitos, et lorsque la mère revient de l'Acropole, Ödipos quitte le foyer familial en compagnie de Hepatitos (p. 121-123). Mais il s'agit là d'une greffe isolée qui n'affecte pas la structure d'ensemble, si ce n'est en accentuant la figure du renversement, cette fois du tragique au comique.

35. Jago, p. 121-128 et 136. Lysistrata, p. 32-33,présente aussi un type de "grande scène ", sur une double page, avec au début de l'album le tableau de la bataille sanglante entre Athéniens et Spartiates sous les murs de l'Acropole, mais a une fonction essentiellement structurelle, servant surtout à introduire la scène parallèle, avec la même perspective et au même endroit, de l'orgie réconciliatrice, p. 114-115, exprimant graphiquement la logique de renversement de l'album.

36. Jago, p. 100.

37. Über Kunst und Alterthum, 1827, où Goethe part justement de la réécriture de son Torquato Tasso par un auteur français pour y voir l'un des symptômes de la constitution d'une « littérature universelle ». 


\section{RÉSUMÉS}

Les bandes dessinées de Ralf König (né en 1960) sont surtout connues depuis ses débuts dans les années 1980 pour leur description humoristique et sensible du milieu homosexuel contemporain. Mais deux albums se détachent de cette tendance, Lysistrata (1987) et Jago (1998), qui reprennent et retravaillent la comédie du même nom d'Aristophane et Othello de Shakespeare. L'article revient sur les relations entre bande dessinée et "grande » littérature, et sur leurs pratiques intertextuelles communes, avant d'analyser les procédés de réécritures auxquels recourt König. Il ne s'en tient pas à l'introduction de la thématique homosexuelle et à des transformations ou des surenchères comiques, visuelles et verbales, mais s'appuie sur la nature théâtrale des textes originaux en jouant avec leurs structures, en redistribuant des motifs empruntés à d'autres pièces, pour aboutir à des créations à la fois riches et personnelles, qui montrent la part que peut prendre la bande dessinée dans le renouvellement de la littérature.

Since he started to write comics in the early 1980's, most books of Ralf König (born 1960) deal with the humourous and moving representation of modern (and male) homosexuality. However, two books do not fit into this frame, Lysistrata (1987) and Jago (1998), in which König reshapes and transforms the comedy of Aristophanes and Shakespeare's Othello. This article first studies the relationship between « higher " literature and comics and shows that both are inclined to intertextual tendencies. The article then analyses König's own literary devices. König not only adds his usual homosexual themes and intensifies comedic aspects by visual and textual means, but also reinterprets theatrical structures and combines motifs from other plays to produce new meanings. This way of playing with literary traditions leads to original work and demonstrates the ability of comic books to renew literature.

Die meisten Comics von Ralf König (geb. 1960) schildern seit seinen Anfängen in den 1980er Jahren auf humorvolle und rührende Weise die schwule Szene. Dabei bilden zwei Bände eine Ausnahme : In Lysistrata (1987) und Jago (1998) wendet sich König von der Gegenwart ab und bearbeitet die gleichnamige Komödie des Aristophanes sowie Shakespeares Othello. Dieser Aufsatz untersucht einleitend die Beziehungen zwischen Comics und "hoher " bzw. ernster Literatur und zeigt, wie sich an beiden eine ähnliche Einstellung zur Intertextualität feststellen lässt, bevor Königs eigene Übertragungs- und Umdeutungsverfahren näher analysiert werden. Er belässt es nämlich nicht bei der Einführung des Themas der Homosexualität und bei der Zuspitzung der Komik in Bild und Dialog, sondern benutzt den dramatischen Charakter seiner Vorlagen, um mit ihren Strukturen zu spielen, und Motive aus anderen Stücken neu zu kombinieren. Diese Arbeit an der literarischen Tradition führt zu komplexen und persönlichen Werken und beweist, wie Comics zur Erneuerung der Literatur beitragen können.

\section{INDEX}

Mots-clés : Aristophane, Shakespeare

\section{AUTEURS}

\section{ÉRIC CHEVREL}

Université Paris IV 